\title{
ASYMPTOTICALLY AUTONOMOUS SEMIFLOWS: CHAIN RECURRENCE AND LYAPUNOV FUNCTIONS
}

\author{
KONSTANTIN MISCHAIKOW, HAL SMITH, AND HORST R. THIEME
}

\begin{abstract}
From the work of C. Conley, it is known that the omega limit set of a precompact orbit of an autonomous semiflow is a chain recurrent set. Here, we improve a result of $L$. Markus by showing that the omega limit set of a solution of an asymptotically autonomous semiflow is a chain recurrent set relative to the limiting autonomous semiflow. In the special case that there is a Lyapunov function for the limiting semiflow, sufficient conditions are given for an omega limit set of the asymptotically autonomous semiflow to be contained in a level set of the Lyapunov function.
\end{abstract}

\section{INTRODUCTION}

In the well-known paper of Markus [Ma], a fundamental result concerning the large time behavior of solutions of asymptotically autonomous ordinary differential equations is obtained. Recall that the nonautonomous system of differential equations in $\mathbf{R}^{n}$

$$
x^{\prime}=f(t, x),
$$

is said to be asymptotically autonomous-with limit equation

$$
y^{\prime}=g(y),
$$

if

$$
f(t, x) \rightarrow g(x), \quad t \rightarrow \infty,
$$

where the convergence is uniform on each compact subset of $\mathbf{R}^{n}$. For simplicity, we assume in this introduction that $f$ and $g$ are continuous functions and that they are locally Lipschitz on $\mathbf{R}^{n}$. The $\omega$-limit set, $\omega\left(t_{0}, x_{0}\right)$, of a bounded solution $x(t)$ of $(0.1)$ on $t \geq t_{0}$ satisfying $x\left(t_{0}\right)=x_{0}$ is defined in the usual way:

$$
\omega\left(t_{0}, x_{0}\right)=\left\{y: y=\lim _{j \rightarrow \infty} x\left(t_{j}\right), \text { for some sequence } t_{j} \rightarrow \infty\right\} .
$$

The result of Markus is the following.

Received by the editors March 29, 1994 and, in revised form, July 5, 1994.

1991 Mathematics Subject Classification. Primary 34C35, 35B40, 58F35.

Key words and phrases. Chain recurrence, asymptotically autonomous semiflow, Lyapunov function.

Research of K. M. funded in part by NSF grant DMS 9101412.

Research of $H$. S. funded in part by NSF grant DMS 9300974.

Research of H. R. T. funded in part by NSF grant DMS 9101979. 
Theorem [Ma]. The $\omega$-limit set $\omega$ of a bounded solution $x(t)$ of $(0.1)$ on $t \geq t_{0}$ is nonempty, compact, and connected. Moreover,

$$
\operatorname{dist}(x(t), \omega) \rightarrow 0, \quad t \rightarrow \infty,
$$

and $\omega$ is invariant under (0.2).

The theorem of Markus says that the $\omega$-limit set of asymptotically autonomous system shares many of the properties known to hold for the $\omega$-limit set of an autonomous system. More recently, Conley [C1, C2] showed that the limit sets of autonomous systems have the property of chain recurrence. A precise definition is given in $\S 1$. Later work of Franke and Selgrade [FS] showed that any nonempty, compact, connected, chain recurrent set, $A$, for an autonomous system is an $\omega$-limit set in the sense that there is a flow on a compact metric space $X$ and $x \in X$ such that the flow restricted to $\omega(x)$ is topologically conjugate to the flow on $A$. Thus, chain recurrence is the property of an $\omega$ limit set that expresses the recurrent behavior of the flow restricted to the limit set.

In this paper we show that an $\omega$-limit set of an asymptotically autonomous system is chain recurrent with respect to the limiting system. In fact, we obtain a more general result which is applicable to delay-differential equations, partial differential equations, and other kinds of finite- and infinite-dimensional dynamical systems. Following the earlier work of the third author [T1], our results are framed in the setting of asymptotically autonomous semiflows on a metric space. This is a natural point of view if one seeks results which are independent of how a dynamical system is generated.

We also obtain a sort of converse result for the special case of ordinary differential equations. Namely, if $A$ is any nonempty, compact, connected, chain recurrent set for $(0.2)$ and $y_{0} \in A$, then there is a smooth function $\psi:[0, \infty) \rightarrow \mathbf{R}^{n}$ such that $\psi(t) \rightarrow 0$ as $t \rightarrow \infty$ and such that the $\omega$-limit set of the solution of

$$
y^{\prime}=g(y)+\psi(t), \quad y(0)=y_{0},
$$

is $A$. This result, together with the previously mentioned one, implies that the property of chain recurrence essentially captures the recurrence properties of the autonomous semiflow restricted to the $\omega$-limit set of an asymptotically autonomous semiflow. It also shows that the trajectories of an asymptotically autonomous system may have a more complicated large-time behavior than the trajectories of the associated limit system (see also [T2]). Finally, in combination with [T1], we use it to describe certain chain recurrent sets for $(0.2)$.

Conley [Cl, Lemma 4.1E] points out an interesting connection between the chain recurrent points of an autonomous flow on a compact metric space and the Lyapunov functions of this flow. By a Lyapunov function we mean a continuous function that is nonincreasing along trajectories. If a point is not chain recurrent with respect to the flow, then there exists a Lyapunov function that is not constant along the whole trajectory through this point. On the other hand, it is well known that the $\omega$-limit set of an autonomous system possessing a Lyapunov function lies in a level set of the function. This property may fail for asymptotically autonomous semiflows, but using a lemma of Ball [B], we can still show the following result which is a generalization to general semiflows of Ball's result for semilinear evolution equations: each number belonging to the 
closed interval of real numbers with lower (upper) limit being the limit inferior (superior) as $t \rightarrow \infty$ of the Lyapunov function evaluated along a solution of the asymptotically autonomous semiflow whose orbit is precompact defines a level set which contains a forward orbit of the autonomous limiting semiflow initiating from a point of the $\omega$-limit set of the asymptotically autonomous system. Several stronger relations of the asymptotically autonomous semiflow to its limiting autonomous semiflow are identified which imply that the closed interval degenerates to a single point. That is, the $\omega$-limit set of the asymptotically autonomous semiflow is contained in a single level set of the Lyapunov function.

This paper is organized as follows. In $\S 1$ the definition of an asymptotically autonomous semiflow is given and sufficient conditions for nonautonomous ordinary and delay differential equations to generate asymptotically autonomous semiflows are provided. Chain recurrence is defined and the $\omega$-limit set of an asymptotically autonomous semiflow is shown to be chain recurrent for the limiting semiflow. The case that the limiting system possesses a Lyapunov function is discussed in $\S 2$. Section 3 contains the statement and proof of the converse result.

The second author would like to acknowledge the support of the Center for Dynamical Systems and Nonlinear Studies at Georgia Institute of Technology during his sabbatical leave.

\section{ASYMPTOTICALLY AUTONOMOUS SEMIFLOWS AND CHAIN RECURRENCE}

Let $(X, d)$ be a metric space. Consider the mapping $\Phi: \Delta \times X \rightarrow X$ on $\Delta=\left\{(t, s): t_{0} \leq s \leq t<\infty\right\}$. $\Phi$ is called a nonautonomous semiflow if it is continuous and satisfies:

(i) $\Phi(s, s, x)=x, s \geq t_{0}$.

(ii) $\boldsymbol{\Phi}(t, s, \boldsymbol{\Phi}(s, r, x))=\Phi(t, r, x), t \geq s \geq r \geq t_{0}$.

The semiflow is called autonomous, if, in addition,

(iii) $\Phi(t+r, s+r, x)=\Phi(t, s, x)$.

Setting $\boldsymbol{\Theta}(t, x)=\Phi\left(t+t_{0}, t_{0}, x\right)$ then the map $\boldsymbol{\Theta}:[0, \infty) \times X \rightarrow X$ will be referred to as an autonomous semiflow. Since $\Phi(t, s, x)=\boldsymbol{\theta}(t-s, x)$, it is sufficient to study $\boldsymbol{\Theta}$. It is continuous and satisfies:

(iv) $\boldsymbol{\theta}(0, x)=x$.

(v) $\boldsymbol{\theta}(t, \boldsymbol{\Theta}(s, x))=\boldsymbol{\Theta}(t+s, x), t, s \geq 0$.

Hereafter, we will always refer to the map $\Theta$ when considering the autonomous semiflow. A subset $A$ of $X$ is said to be positively invariant for $\Theta$ if for all $a \in A$ and $t \geq 0, \Theta(t, a) \in A . A$ is called invariant if it is positively invariant and for all $a \in A$ and $t \geq 0$ there exists $b \in A$ such that $\boldsymbol{\Theta}(t, b)=a$.

A nonautonomous semiflow $\Phi$ on $X$ is called asymptotically autonomouswith limit semiflow $\boldsymbol{\theta}$ - if $\boldsymbol{\theta}$ is an autonomous semiflow on $X$ and

$$
\boldsymbol{\Phi}\left(t_{j}+s_{j}, s_{j}, x_{j}\right) \rightarrow \boldsymbol{\theta}(t, x), \quad j \rightarrow \infty,
$$

for any three sequences $t_{j} \rightarrow t, s_{j} \rightarrow \infty, x_{j} \rightarrow x, j \rightarrow \infty$, with $x, x_{j} \in$ $X, 0 \leq t, t_{j}<\infty$, and $s_{j} \geq t_{0}$. For systems of ordinary differential equations, well-known modes of convergence of the nonautonomous vector field to the autonomous vector field imply that the nonautonomous semiflow is asymptotically autonomous in the sense defined above. See, e.g., [Ma], [S, p. 184]. 
Consider the systems of ordinary differential equations $x^{\prime}=f(t, x)$ and $y^{\prime}=g(y)$ on $\mathbf{R}^{n}$. We assume that $f$ and $g$ are continuous and that initial value problems for each system have unique solutions defined for all future times. Denote by $\Phi\left(t, s, x_{0}\right)$ the solution $x(t)$ of the first system satisfying $x(s)=x_{0}$ and denote by $\Theta\left(t, x_{0}\right)$ the solution $y(t)$ of the second system satisfying $y(0)=x_{0}$.

Proposition 1.1. $\Phi$ is asymptotically autonomous with limit semiflow $\Theta$ if one of the two conditions (A) or (B) is satisfied:

(A) $f(t, x) \rightarrow g(x), t \rightarrow \infty$, uniformly on compact subsets of $\mathbf{R}^{n}$.

(B) $g$ is locally Lipschitz and for each compact subset $K \subseteq \mathbf{R}^{n}$ there is a function $\mu_{K}:[0, \infty) \rightarrow[0, \infty)$ satisfying $\mu_{K}(t) \rightarrow 0, t \rightarrow \infty$, and

$$
\left|\int_{t}^{t+\sigma}[f(s, x)-g(x)] d s\right| \leq \mu_{K}(t)
$$

for every $(x, \sigma) \in K \times[0,1]$ and $t \geq 0$.

Proof. (A) Suppose $x_{j} \rightarrow x_{0}, t_{j} \rightarrow t_{0}, s_{j} \rightarrow \infty$ as $j \rightarrow \infty$. Let $T>0$ be such that $t_{j} \leq T, j \geq 0$, and define

$$
u_{j}(t)=\Phi\left(t+s_{j}, s_{j}, x_{j}\right) \text { and } u(t)=\boldsymbol{\Theta}\left(t, x_{0}\right)
$$

for $0 \leq t \leq T$. Since $u_{j}(t)$ satisfies

$$
x^{\prime}=f\left(t+s_{j}, x\right), \quad x(0)=x_{j}
$$

and $f\left(t+s_{j}, x\right) \rightarrow g(x), j \rightarrow \infty$, uniformly on compact subsets of $\mathbf{R}^{n+1}$, a standard result in the theory of ordinary differential equations [H1, Chapter 1, Lemma 3.1] implies that $u_{j}(t) \rightarrow u(t), j \rightarrow \infty$, uniformly on $[0, T]$. Consequently,

$$
\Phi\left(t_{j}+s_{j}, s_{j}, x_{j}\right) \rightarrow \Theta\left(t_{0}, x_{0}\right) \text { as } j \rightarrow \infty .
$$

(B) Let $u_{j}$ be as above and $v_{j}(t)=\boldsymbol{\Theta}\left(t, x_{j}\right)$. As $v_{j} \rightarrow u$ uniformly on $[0, T]$, there exist a compact set $K$ and $\varepsilon>0$ such that $K$ contains $\bigcup\left\{y:\left|y-v_{j}(t)\right|<\varepsilon\right\}$ where the union is taken over all $j$ and all $t \in[0, T]$. Let $L$ be a Lipschitz constant for $g$ on $K$ and choose $\delta>0$ such that $\delta e^{L T}<\varepsilon$. By our assumption, there exists a function $\mu_{K}(t)$ on $t \geq 0$ satisfying $\mu_{K}(t) \rightarrow 0, t \rightarrow \infty$, and

$$
\left|\int_{t}^{t+\sigma}[f(s, x)-g(x)] d s\right| \leq \mu_{K}(t)
$$

for $x \in K, 0 \leq \sigma \leq T$. Choose $S>0$ such that $\mu_{K}(s)<\delta$ for $s>S$. If $s_{j}>S$, then

$$
\begin{aligned}
\left|u_{j}(t)-v_{j}(t)\right| \leq & \left|\int_{s_{j}}^{s_{j}+t}\left[f\left(\eta, u_{j}\left(\eta-s_{j}\right)\right)-g\left(u_{j}\left(\eta-s_{j}\right)\right)\right] d \eta\right| \\
& +\int_{0}^{t}\left|g\left(u_{j}(\eta)\right)-g\left(v_{j}(\eta)\right)\right| d \eta \\
\leq & \mu_{K}\left(s_{j}\right)+L \int_{0}^{t}\left|u_{j}(\eta)-v_{j}(\eta)\right| d \eta .
\end{aligned}
$$


Gronwall's inequality implies that

$$
\left|u_{j}(t)-v_{j}(t)\right| \leq \mu_{K}\left(s_{j}\right) e^{L t} \leq \delta e^{L T}<\varepsilon, \quad 0 \leq t \leq T .
$$

It follows that $u_{j}(t) \in K$ for all $t \in[0, T]$ if $j$ is such that $s_{j}>S$ and that $u_{j} \rightarrow u, j \rightarrow \infty$, uniformly. As in part (A), this completes the proof.

A similar result holds for functional differential equations with bounded delays. Let $C=C\left([-r, 0], \mathbf{R}^{n}\right), t>0$, and $f: \mathbf{R} \times C \rightarrow \mathbf{R}^{n}, g: C \rightarrow \mathbf{R}^{n}$ be continuous. Consider the equations

$$
x^{\prime}(t)=f\left(t, x_{t}\right) \text { and } y^{\prime}(t)=g\left(y_{t}\right) .
$$

As usual, $x_{t} \in C$ is defined by $x_{t}(s)=x(t+s),-r \leq s \leq 0$. We assume that initial value problems have unique solutions defined for all future time. If for every compact subset $K$ of $C$ there is a neighborhood $V$ of $K$ such that

$$
f(t, \varphi) \rightarrow g(\varphi), \quad t \rightarrow \infty,
$$

uniformly for $\varphi \in V$, then the semiflow $\Phi(t, s, \varphi)=x_{t}(s, \varphi)$ is asymptotically autonomous with limit semiflow $\Theta(t, \varphi) \equiv y_{t}(0, \varphi)$. The proof uses [HV, Chapter 2, Theorem 2.2] as in the proof of (A) of the proposition.

Using the same ideas as in the proof of Proposition 1.1(A) one can also treat semilinear evolution systems

$$
\frac{d u}{d t}=A u+f(t, u),
$$

following the work of Ball [B]. Assuming that $A$ generates a $C_{0}$-semigroup $T$ on the Banach space $X$ and $f:\left[t_{0}, \infty\right) \times X \rightarrow X$ is continuous, we consider mild solutions of the system, that is, continuous solutions of the variation of constants formula

$$
u(t)=T(t-r) u(r)+\int_{r}^{t} T(t-s) f(s, u(s)) d s .
$$

It will be assumed that mild solutions exist, are uniquely determined by $u(r)$ and extend to $t \geq r$. Consider also the time-independent system

$$
\frac{d v}{d t}=A v+g(v)
$$

and the mild formulation

$$
v(t)=T(t) v(0)+\int_{0}^{t} T(t-s) g(v(s)) d s .
$$

Assume that $g: X \rightarrow X$ is Lipschitz on each bounded subset of $X$ and that mild solutions extend to $t \geq 0$. If

$$
f(t, u) \rightarrow g(u), \quad t \rightarrow \infty,
$$

uniformly on bounded subsets of $X$, then the nonautonomous semiflow $\Phi(t, r, u(r))=u(t)$ is asymptotically autonomous with limit semiflow

$$
\boldsymbol{\Theta}(t, v(0))=v(t) .
$$

If $\Phi$ is a nonautonomous semiflow on $X$ and $(s, x) \in\left[t_{0}, \infty\right) \times X$, then the forward orbit of $\Phi$ through $(s, x)$ is defined to be

$$
\mathscr{\mathcal { O }}_{\Phi}(s, x)=\{\Phi(t, s, x): t \geq s\} \subset X .
$$


If $\mathscr{O}_{\Phi}(s, x)$ has compact closure in $X$, then the $\omega$-limit set of $(s, x)$ (or of $\left.\mathscr{Q}_{\Phi}(s, x)\right)$ is defined by

$$
\omega_{\Phi}(s, x)=\bigcap_{\tau \geq s} \overline{\{\Phi(t, s, x): t \geq \tau\}}
$$

where, for a subset $A$ of $X, \bar{A}$ denotes the closure of $A$ in $X$. In other words, $y \in \omega_{\Phi}(s, x)$ if there is a sequence $t_{j} \rightarrow \infty, t_{j} \geq s$, such that $\Phi\left(t_{j}, s, x\right) \rightarrow$ $y, j \rightarrow \infty$.

In the case of an autonomous semiflow $\boldsymbol{\theta}$, the $\omega$-limit set is independent of $s$ and hence we denote it by $\omega_{\Theta}(x)$ :

$$
\omega_{\Theta}(x)=\bigcap_{\tau \geq 0} \overline{\{\boldsymbol{\theta}(t, x): t \geq \tau\}} .
$$

It has the following well-known properties [H2].

Proposition 1.2. Let $\Theta$ be an autonomous semiflow and suppose that $\mathscr{O}_{\Theta}(x)=$ $\{\boldsymbol{\Theta}(t, x): t \geq 0\}$ has compact closure in $X$. Then $\omega_{\Theta}(x)$ has the following properties:

(a) $\omega_{\Theta}(x)$ is nonempty, compact, and connected.

(b) $\omega_{\Theta}(x)$ is invariant.

(c) $\omega_{\Theta}(x)$ attracts $\boldsymbol{\Theta}(t, x)$ :

$$
\operatorname{dist}_{X}\left(\Theta(t, x), \omega_{\Theta}(x)\right) \rightarrow 0, \quad t \rightarrow \infty .
$$

In addition to the above properties, $\omega_{\Theta}(x)$ consists of chain recurrent points for the semiflow $\boldsymbol{\theta}$, restricted to the compact invariant set $\omega_{\Theta}(x)$. The notion of chain recurrence and the above-mentioned result are due to Conley [C1, C2]. We give a brief description of the basic ideas.

Definition 1.3 (Chain recurrence). Let $A$ be a nonempty positively invariant subset of $X$ and $x, y \in A$. For $\varepsilon>0, t>0$, an $(\varepsilon, t)$-chain from $x$ to $y$ (in $A$ ) is a sequence $\left\{x=x_{1}, x_{2}, \ldots, x_{n+1}=y ; t_{1}, t_{2}, \ldots, t_{n}\right\}$ of points $x_{i} \in A$ and times $t_{i} \geq t$ such that $d\left(\Theta\left(t_{i}, x_{i}\right), x_{i+1}\right)<\varepsilon, i=1,2, \ldots, n$. A point $x \in A$ is called chain recurrent (in $A$ ) if for every $\varepsilon>0, t>0$ there is an $(\varepsilon, t)$-chain from $x$ to $x$ in $A$. The set $A$ is said to be chain recurrent if every point in $A$ is chain recurrent in $A$. We will be primarily interested in the case that $A$ is compact, connected and invariant.

The following lemma rephrases Theorem 3.3 in [C1]. For the convenience of the reader Conley's proof is repeated below.

Lemma 1.4. Let $A$ be connected and chain recurrent. Then, for any $x, y \in$ $A, \varepsilon>0, T>0$, there exists an $(\varepsilon, T)$-chain from $x$ to $y$.

Proof (Conley). As $A$ is connected, for any $\delta>0$, there exist $z_{1}, \ldots, z_{n+1} \in$ $A$, with $x=z_{1}$ and $z_{n+1}=y$ such that

$$
d\left(z_{j}, z_{j+1}\right)<\delta, \quad j=1, \ldots, n .
$$

Let $\varepsilon>0$ and $\delta=\varepsilon / 2$. Fix $j \in\{1, \ldots, n\}$. As $z_{j} \in A$ and $A$ is chain recurrent, there exists a $(\delta, T)$-chain from $z_{j}$ to $z_{j}$,

$$
z_{j}=x_{1}, \ldots, x_{m+1}=z_{j}, \quad x_{k} \in A \text {. }
$$


Then

$$
z_{j}=x_{1}, \ldots, x_{m}, \tilde{x}_{m+1}=z_{j+1}
$$

is an $(\varepsilon, t)$-chain from $z_{j}$ to $z_{j+1}$. Combining all $(\varepsilon, T)$-chains from $z_{j}$ for $z_{j+1}, j=1, \ldots, n$, yields an $(\varepsilon, T)$-chain from $x=z_{1}$ to $y=z_{n+1}$.

The following result is due to Conley [C2] in the case of a flow. We are unaware of a reference for the result in the case of a semiflow, so we include a proof (following one given by Robinson [R] for mappings).

Proposition 1.5. Let the hypotheses of Proposition 1.2 hold. Then $\omega_{\Theta}(x)$ is chain recurrent.

Our proof uses the following intuitive result.

Lemma 1.6. Let $T>0$ and $\mathscr{O}_{\Theta}^{T}(x)=\{\Theta(t, x): t \geq T\}$. Given $y \in \omega_{\Theta}(x)$ and $\varepsilon>0, t_{0}>0$, there exists an $\left(\varepsilon, t_{0}\right)$-chain

$$
\left\{y=y_{1}, y_{2}, \ldots, y_{l}, y_{l+1}=y ; t_{1}, t_{2}, \ldots, t_{l}\right\}
$$

such that $y_{i} \in \mathscr{O}_{\boldsymbol{\Theta}}^{T}(x)$ for $i=2, \ldots, l, t_{i}=t_{0}, 1 \leq i \leq l-1, t_{0} \leq t_{l}<2 t_{0}$.

Proof. Suppose that $y=\lim _{n \rightarrow \infty} \Theta\left(s_{n}, x\right)$ where $s_{n} \rightarrow \infty, n \rightarrow \infty$. Choose $n$ such that $s_{n}>T$ and $d\left(\Theta\left(s_{n}+t, x\right), \Theta(t, y)\right)<\varepsilon$ for $0 \leq t \leq t_{0}$. Set $y_{1}=y$ and $y_{2}=\boldsymbol{\Theta}\left(s_{n}+t_{0}, x\right)$ and $t_{1}=t_{0}$. Then

$$
d\left(\boldsymbol{\Theta}\left(t_{1}, y_{1}\right), y_{2}\right)=d\left(\boldsymbol{\Theta}\left(t_{0}, y\right), \boldsymbol{\Theta}\left(s_{n}+t_{0}, x\right)\right)<\varepsilon .
$$

Choose $m$ such that $s_{m}>s_{n}+2 t_{0}$ and $d\left(\Theta\left(s_{m}, x\right), y\right)<\varepsilon$. Let $k \geq 1$ be such that $s_{m}-s_{n}-t_{0}=k t_{0}+r$ for some $r, 0 \leq r<t_{0}$. Let $y_{3}=$ $\boldsymbol{\Theta}\left(s_{n}+2 t_{0}, x\right), y_{4}=\boldsymbol{\Theta}\left(s_{n}+3 t_{0}, x\right), \ldots, y_{k+1}=\boldsymbol{\Theta}\left(s_{n}+k t_{0}, x\right), y_{k+2}=y$, and $t_{i}=t_{0}, 1 \leq i \leq k, t_{k+1}=t_{0}+r$. Then $d\left(\boldsymbol{\theta}\left(t_{i}, y_{i}\right), y_{i+1}\right)=0, i=1,2, \ldots, k$, and $d\left(\boldsymbol{\Theta}\left(\bar{t}_{k+1}, y_{k+1}\right), y_{k+2}\right)=d\left(\boldsymbol{\Theta}\left(s_{m}, x\right), y\right)<\varepsilon$.

Proof of Proposition 1.5. Let $y \in \omega_{\theta}(x)$ and $\varepsilon>0, t_{0}>0$. By Lemma 1.6, for each $n=1,2, \ldots$, there exists a $\left(\frac{1}{n}, t_{0}\right)$-chain belonging to $\mathscr{O}_{\Theta}^{n}(x)$ having the properties described in Lemma 1.6 with $\varepsilon=1 / n$. Let $y_{i}^{n}, 1 \leq i \leq l_{n}+1$, be the points of the $\left(\frac{1}{n}, t_{0}\right)$-chain, $t_{i}^{n}$ be the times, $1 \leq i \leq l_{n} \quad\left(t_{i}^{n}=t_{0}, 1 \leq i \leq\right.$ $\left.l_{n}-1, t_{0} \leq t_{l_{n}}^{n}<2 t_{0}\right)$ and set $C^{n}=\left\{y_{i}^{n}: 1 \leq i \leq l_{n}\right\}$.

Since $C^{n} \subset \overline{\mathscr{O}_{\Theta}(x)}$ and $\overline{\mathscr{O}_{\Theta}(x)}$ is compact in $X$, by passing to a subsequence, if necessary, we can assume that $C^{n} \rightarrow C$ as $n \rightarrow \infty$ in the Hausdorff metric on the space of closed subsets of $\overline{\mathscr{\sigma}_{\Theta}(x)}$, where $C$ is a compact subset of $\overline{\mathscr{\sigma}_{\Theta}(x)}$. In fact, as $y \in C^{n} \subset \mathscr{O}_{\boldsymbol{\Theta}}^{n}(x)$ it follows that $y \in C \subset \omega_{\Theta}(x)$.

Choose $\delta, 0<\delta<\varepsilon / 3$, such that whenever $z_{1}, z_{2} \in \overline{\mathscr{\sigma}_{\Theta}(x)}$ and $d\left(z_{1}, z_{2}\right)$ $<\delta$ then $d\left(\Theta\left(t, z_{1}\right), \Theta\left(t, z_{2}\right)\right)<\varepsilon / 3$ for $0 \leq t \leq 2 t_{0}$. Fix $n$ such that $1 / n<\varepsilon / 3$ and $D\left(C^{n}, C\right)<\delta$, where $D$ denotes the Hausdorff metric. Drop the superscript $n$ on $y_{i}^{n}$ and $t_{i}^{n}$. Then we have

$$
d\left(\boldsymbol{\Theta}\left(t_{1}, y_{1}\right), y_{2}\right)=d\left(\boldsymbol{\Theta}\left(t_{0}, y\right), y_{2}\right)<\frac{1}{n}<\frac{\varepsilon}{3} .
$$

Set $z_{1}=y$ and choose $z_{2} \in C$ such that $d\left(z_{2}, y_{2}\right)<\delta<\varepsilon / 3$. Then

$$
\begin{aligned}
& d\left(\boldsymbol{\Theta}\left(t_{1}, z_{1}\right), z_{2}\right)=d\left(\boldsymbol{\Theta}\left(t_{0}, y\right), z_{2}\right) \\
& \quad \leq d\left(\boldsymbol{\Theta}\left(t_{0}, y\right), y_{2}\right)+d\left(y_{2}, z_{2}\right)<2 \varepsilon / 3 .
\end{aligned}
$$


Since $d\left(z_{2}, y_{2}\right)<\delta$, it follows that

$$
d\left(\boldsymbol{\Theta}\left(t_{2}, z_{2}\right), \boldsymbol{\Theta}\left(t_{2}, y_{2}\right)\right)<\varepsilon / 3 .
$$

Choose $z_{3} \in C$ such that $d\left(z_{3}, y_{3}\right)<\delta<\varepsilon / 3$. Then

$$
\begin{aligned}
d\left(\boldsymbol{\Theta}\left(t_{2}, z_{2}\right), z_{3}\right) & \leq d\left(\boldsymbol{\Theta}\left(t_{2}, z_{2}\right), \boldsymbol{\Theta}\left(t_{2}, y_{2}\right)\right)+d\left(\boldsymbol{\Theta}\left(t_{2}, y_{2}\right), y_{3}\right)+d\left(y_{3}, z_{3}\right) \\
& <\frac{\varepsilon}{3}+\frac{1}{n}+\delta<\varepsilon .
\end{aligned}
$$

Furthermore, $d\left(z_{3}, y_{3}\right)<\delta$ implies

$$
d\left(\boldsymbol{\Theta}\left(t_{3}, z_{3}\right), \boldsymbol{\Theta}\left(t_{3}, y_{3}\right)\right)<\varepsilon / 3,
$$

so we choose $z_{4} \in C$ such that $d\left(z_{4}, y_{4}\right)<\delta<\varepsilon / 3$. As above, $d\left(\boldsymbol{\Theta}\left(t_{3}, z_{3}\right), z_{4}\right)$ $<\varepsilon$. Clearly, we may continue in this way, finding $z_{i} \in C$ with $d\left(z_{i}, y_{i}\right)<\delta$, $i=1,2, \ldots, l=l_{n}$ and $t_{i}, i=1,2, \ldots, l-1$, such that $d\left(\Theta\left(t_{i}, z_{i}\right), z_{i+1}\right)<$ $\varepsilon$ for $i=1,2, \ldots, l-1$. Since $d\left(z_{l}, y_{l}\right)<\delta$, we may conclude that $d\left(\boldsymbol{\Theta}\left(t_{l}, z_{l}\right), \boldsymbol{\Theta}\left(t_{l}, y_{l}\right)\right)<\varepsilon / 3$. Set $z_{l+1}=y$ and observe that

$$
\begin{aligned}
d\left(\boldsymbol{\Theta}\left(t_{l}, z_{l}\right), z_{l+1}\right) & \leq d\left(\boldsymbol{\Theta}\left(t_{l}, z_{l}\right), \boldsymbol{\Theta}\left(t_{l}, y_{l}\right)\right)+d\left(\boldsymbol{\Theta}\left(t_{l}, y_{l}\right), y\right) \\
& <\frac{\varepsilon}{3}+\frac{1}{n}<\frac{2}{3} \varepsilon .
\end{aligned}
$$

We have constructed an $\left(\varepsilon, t_{0}\right)$-chain in $C \subset \omega_{\Theta}(x)$ joining the point $y$ to itself. Since $y \in \omega_{\Theta}(x), \varepsilon>0, t_{0}>0$ were arbitrary, it follows that $\omega_{\Theta}(x)$ is chain recurrent. This concludes our proof.

In [T1] it is observed that if $\Phi$ is a nonautonomous semiflow which is asymptotically autonomous with limit semiflow $\theta$, then $\Phi$ and $\theta$ can be imbedded in a single autonomous semiflow $\Psi$ on the larger metric space $Z=\left[t_{0}, \infty\right] \times X$, where $\left[t_{0}, \infty\right]$ is compactified in the usual way, by

$$
\Psi(t,(s, x))= \begin{cases}(t+s, \boldsymbol{\Phi}(t+s, s, x)), & t_{0} \leq s<\infty, \\ (\infty, \boldsymbol{\Theta}(t, x)), & s=\infty\end{cases}
$$

for $t \geq 0$. More precisely, $\Psi:[0, \infty) \times Z \rightarrow Z$ is continuous and enjoys the property of being a semiflow on $Z$.

There are several classical techniques for converting nonautonomous systems into autonomous ones. Including time as a state variable, as we have just done, is a classical way of reducing the uniqueness and existence proof for nonautonomous ordinary differential equations to the autonomous case [NS]. For general nonautonomous equations it is an unproductive way to study the large time behavior of solutions, but for an asymptotically autonomous system it offers a convenient reduction to the autonomous case which is simpler than the theory of skew product flows which has been suggested by Miller and Sell [Mi, MS1, MS2, Se1, Se2] for general nonautonomous ordinary differential equations, integral equations, and delay differential equations. Skew product flows give rise to autonomous systems on a larger state space that typically includes a space of vector fields, or their analogs, and subtle topological considerations are required to obtain a suitable topology on the space that guarantees continuity. These complications are aggravated when these ideas are extended to general processes as proposed by Dafermos [D] and LaSalle [L]. See [H2, Sa] for more on skew product flows and further references. 
It will be convenient at a later point to have an explicit metric for $Z$. We take the metric $\rho((s, x),(t, y))=|h(s)-h(t)|+d(x, y)$ where $h:\left[t_{0}, \infty\right] \rightarrow[0,1]$ is the map $h(t)=\left(t-t_{0}\right) /\left(1+\left(t-t_{0}\right)\right), t<\infty$, and $h(\infty)=1$.

If $(s, x) \in Z$ and $s<\infty$, then there is a simple relationship between $\omega_{\Psi}(s, x)$ and $\omega_{\Phi}(s, x)$ which is contained in the following result.

Lemma 1.7 [T1]. Let $\Phi$ be an asymptotically autonomous semiflow with limit semiflow $\boldsymbol{\Theta}$, and let $\mathscr{O}_{\Phi}(s, x)$ have compact closure in $X$. Then $\mathscr{O}_{\Psi}(s, x)$ has compact closure in $Z$ and

$$
\{\infty\} \times \omega_{\Phi}(s, x)=\omega_{\Psi}(s, x) .
$$

Note that since $\Phi$ is a nonautonomous semiflow, we must include the "starting time" $s$ in the $\omega$-limit set notation, $\omega_{\Phi}(s, x)$, whereas $\Psi$ is an autonomous semiflow starting at $t=0$ at the state $(s, x) \in Z$ so the notation, $\omega_{\Psi}(s, x)$, reflects that $s$ is part of the state $(s, x)$ for the semiflow $\Psi$.

Propositions 1.2 and 1.5, applied to the semiflow $\Psi$, together with Lemma 1.7 and the fact that $\Psi(t,(\infty, y))=(\infty, \boldsymbol{\Theta}(t, y))$ for all $y \in \omega_{\Phi}(s, x)$ yield the following result, all but the last of which appears in [T1]. For ordinary differential equations, Markus [Ma] proved (a), (b), and (c).

Theorem 1.8. Let $\Phi$ be an asymptotically autonomous semiflow with limit semiflow $\boldsymbol{\Theta}$, and let $\mathcal{O}_{\Phi}(s, x)$ have compact closure in $X$. Then $\omega=\omega_{\Phi}(s, x)$ has the following properties:

(a) $\omega$ is nonempty, compact, and connected.

(b) $\omega$ is invariant for the semiflow $\boldsymbol{\Theta}$ :

$$
\boldsymbol{\Theta}(t, \omega)=\omega \text { for each } t \geq 0 .
$$

(c) $\omega$ attracts $\Phi(t, s, x)$ :

$$
\operatorname{dist}_{X}(\Phi(t, s, x), \omega) \rightarrow 0, \quad t \rightarrow \infty .
$$

(d) $\omega$ is chain recurrent for $\boldsymbol{\Theta}$.

Proof. By Lemma 1.7, $\mathscr{O}_{\Psi}(s, x)$ has compact closure in $Z$ and, consequently, by Proposition 1.2, $\omega_{\Psi}(s, x)$ is nonempty, compact, and connected. By (1.1), $\omega$ has these properties. Since $\omega_{\Psi}(s, x)=\{\infty\} \times \omega$ is invariant for $\Psi$, it follows that $\omega$ is invariant for $\boldsymbol{\Theta}$. Similarly, $\{\infty\} \times \omega$ is chain recurrent for $\Psi$, by Proposition 1.5, and this immediately implies that $\omega$ is chain recurrent for $\boldsymbol{\Theta}$. Finally, since

$$
\operatorname{dist}_{Z}(\Psi(t,(s, x)),\{\infty\} \times \omega)=\frac{1}{1+\left(t+s-t_{0}\right)}+\operatorname{dist}_{X}(\Phi(t, s, x), \omega)
$$

tends to zero as $t \rightarrow \infty$, by Proposition 1.2(iii), it follows that

$$
\operatorname{dist}_{X}(\Phi(t, s, x), \omega) \rightarrow 0 \text { as } t \rightarrow \infty \text {. }
$$

Assertions (a) and (c) of Theorem 1.8 hold without the assumption that $\Phi$ is asymptotically autonomous. Assertion (b) was established by Markus [Ma] for ordinary differential equations and by Thieme [T1] in the present context. Assertion (d) appears to be new, if not surprising. It implies, for example, that the compact, connected invariant set depicted in Figure 1 cannot be the omega 


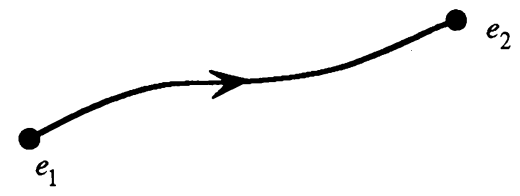

FIGURE 1. An invariant set consisting of two equilibria and a connecting orbit as above is not the omega limit set of an asymptotically autonomous semiflow.

limit set of an asymptotically autonomous semiflow since it fails to be chain recurrent.

As noted in [T1], a consequence of the invariance of $\omega$ for the semiflow $\theta$ is that there is a full $\theta$-orbit through each point $y \in \omega$ which belongs to $\omega$. More precisely, there exists a continuous map $\eta: \mathbf{R} \rightarrow \omega$ satisfying $\eta(0)=y$ and $\boldsymbol{\Theta}(t, \eta(s))=\eta(t+s)$ for all $t \geq 0, s \in \mathbf{R}$.

Theorem 1.8 states that $\omega$-limit sets of the asymptotically autonomous semiflow $\Phi$, with limit semiflow $\boldsymbol{\theta}$, belong to the class of compact, connected subsets of $X$ which are invariant and chain recurrent for $\Theta$. It is natural to ask whether any subset of $X$ with these properties is an $\omega$-limit set of some asymptotically autonomous semiflow $\Phi$ having $\Theta$ as an autonomous limit semiflow. In a later section we show that this is indeed the case for systems of ordinary differential equations.

\section{ASYMPTOTICALLY AUTONOMOUS SEMIFLOWS AND LYAPUNOV FUNCTIONS}

Let $(X, d)$ be a metric space and $\Theta:[0, \infty) \times X \rightarrow X$ be an autonomous semiflow on $X$.

Definition 2.1. $\varphi: X \rightarrow \mathbf{R}$ is called a Lyapunov function (for the semiflow $\boldsymbol{\theta}$ ) if the following hold:

(i) $\varphi$ is continuous.

(ii) $\varphi$ decreases along forward orbits; i.e., $\varphi(\Theta(t, x))$ is a nonincreasing function of $t \geq 0$.

We consider an asymptotically autonomous semiflow $\Phi$ on $X$ with limitsemiflow $\boldsymbol{\theta}$. $\Phi$ and $\boldsymbol{\theta}$ are embedded into the autonomous semiflow $\Psi$ on $\left[t_{0}, \infty\right] \times X$. The following result is then a consequence of Theorem 2.3 in Ball [B].

Theorem 2.2. Let $\varphi$ be a Lyapunov function for $\boldsymbol{\Theta}$, and let the forward $\Phi$-orbit starting at $(s, x)$ be precompact. Further, let

$$
\beta_{\infty}=\liminf _{t \rightarrow \infty} \varphi(\Phi(t, s, x)), \quad \beta^{\infty}=\limsup _{t \rightarrow \infty} \varphi(\Phi(t, s, x)) .
$$

Then, for any $\beta \in\left[\beta_{\infty}, \beta^{\infty}\right]$, there exists some $y \in \omega_{\Phi}(s, x)$ such that $\varphi(\Theta(t, y))=\beta$ for all $t \geq 0$.

Proof. We introduce the functional $\psi(s, x)=\varphi(x)$ on the metric space $\left[s_{0}, \infty\right)$ $\times X$. According to Theorem 2.3 in [B] we have to show that, for

$$
f(t)=\psi(\Psi(t,(s, x)))=\varphi(\Phi(t+s, s, x)),
$$

we have

$$
\liminf _{t \rightarrow \infty}(f(t)-f(t+\tau)) \geq 0, \quad \forall \tau \geq 0
$$


We suppose that (2.1) does not hold. Then there exists some $\varepsilon>0, \tau>0$ and a sequence $t_{j} \rightarrow \infty, j \rightarrow \infty$, such that

$$
\limsup _{j \rightarrow \infty}\left(f\left(t_{j}\right)-f\left(t_{j}+\tau\right)\right)<-\varepsilon .
$$

Since the forward orbit starting at $(s, x)$ is precompact, after choosing another subsequence, we can assume that there exists some $y \in X$ such that $\Phi\left(t_{j}+s, s, x\right) \rightarrow y, j \rightarrow \infty$. Hence

$$
\begin{aligned}
-\varepsilon & >\limsup _{j \rightarrow \infty}\left(\varphi\left(\Phi\left(t_{j}+s, s, x\right)\right)-\varphi\left(\Phi\left(t_{j}+\tau+s, s, x\right)\right)\right) \\
& =\limsup _{j \rightarrow \infty}\left(\varphi\left(\Phi\left(t_{j}+s, s, x\right)\right)-\varphi\left(\Phi\left(t_{j}+\tau+s, t_{j}+s, \Phi\left(t_{j}+s, s, x\right)\right)\right)\right) .
\end{aligned}
$$

As $\Phi$ is asymptotically autonomous with limit semiflow $\theta$ and $\phi$ is continuous, we have

$$
-\varepsilon>\varphi(y)-\varphi(\Theta(\tau, y)) \geq 0,
$$

because $\varphi$ is a Lyapunov function for $\Theta$. The contradiction proves (2.1) and the theorem.

Corollary 2.3. Let $\varphi$ be a Lyapunov function for $\boldsymbol{\Theta}$, and let the forward $\boldsymbol{\Phi}$-orbit starting at $(r, x)$ be precompact. Further assume that any compact subset $K$ of $X$ contains at most countably many forward 8 -orbits on which $\varphi$ is constant. Then there exists some $\beta \in \mathbf{R}$ such that $\varphi(y)=\beta$ for any $y \in \omega_{\Phi}(r, x)$. In particular, there exists a full 8 -orbit $\theta$ through any $y \in \omega_{\Phi}(r, x)$ such that $\varphi(\theta(t))=\beta$ for all $t \in \mathbf{R}$.

The assumption that there are only countably many forward orbits on which $\varphi$ is constant is rather restrictive. We aim at dropping it at the expense of requiring that $\varphi$ is Lipschitz on compact subsets and that the convergence of the asymptotically autonomous semiflow to its limit semiflow is sufficiently fast.

Definition 2.4. Let $\eta:(0, \infty) \rightarrow \mathbf{R}$ be a monotone decreasing function with $\eta(t) \rightarrow 0, t \rightarrow \infty$. An asymptotically autonomous semiflow $\Phi$ with limit semiflow $\boldsymbol{\theta}$ is called $\eta$-asymptotically autonomous if, for any compact set $K$ in $X$, there exist some $s_{K}>0, \varepsilon_{K}>0$ such that

$$
d(\Phi(t+s, s, x), \boldsymbol{\Theta}(t, x)) \leq \eta(s)-\eta(t+s), \quad s \geq s_{K}, 0 \leq t \leq \varepsilon_{K}, x \in K .
$$

Theorem 2.5. Let $\Phi$ be $\eta$-asymptotically autonomous for an appropriate function $\eta$. Let the $\Phi$-orbit starting at the point $(r, x)$ be precompact and $\varphi$ be a Lyapunov function for the limit semiflow $\Theta$ such that $\varphi$ is Lipschitz on compact subsets of $X$. Then there exists some $\beta \in \mathbf{R}$ such that $\varphi(y)=\beta$ for all $y \in \omega_{\Phi}(r, x)$. In particular, there exists a full $\boldsymbol{\theta}$-orbit $\theta$ through any $y \in \omega_{\Phi}(r, x)$ such that $\varphi(\theta(t))=\beta$ for all $t \in \mathbf{R}$.

Proof. Let $K$ be the closure of the forward $\Phi$-orbit starting at $(r, x)$. Now choose $\eta, \varepsilon_{K}, s_{K}$ according to Definitions 2.4. Let $\Lambda>0$ be the Lipschitz constant of $\varphi$ on $K$. We define

$$
\psi(s, x)=\varphi(x)+\Lambda \eta(s), \quad s \geq s_{K}, x \in X .
$$

Claim 1. $\psi(\Psi(t,(s, y))) \leq \psi(s, y)$ for $s \geq s_{K}, y \in K, 0 \leq t \leq \varepsilon_{K}$. 
Proof of Claim 1.

$$
\begin{aligned}
& \psi(s, y)-\psi(\Psi(t,(s, y))) \geq \varphi(y)+\Lambda \eta(s)-\varphi(\Phi(t+s, s, y))-\Lambda \eta(t+s) \\
& \quad \geq \varphi(\Theta(t, y))-\varphi(\Phi(t+s, s, y))+\Lambda(\eta(s)-\eta(t+s)) \\
& \quad \geq \Lambda(\eta(s)-\eta(t+s)-d(\Theta(t, y), \Phi(t+s, s, y))) \\
& \quad \geq 0 .
\end{aligned}
$$

Claim 2. $\psi(\Psi(t,(r, x))) \leq \psi(\Psi(s,(r, x)))$ for $t \geq s \geq s_{K}$.

Notice that $\left[s_{K}, \infty\right] \times K$ contains $\Psi(t,(r, y))$ for $t \geq s_{K}-r, t \geq 0$. Claim 2 now follows from Claim 1 via the semiflow property of $\Psi$ in a standard fashion.

By Claim 2 we have that $\psi(\Psi(t,(r, x))) \rightarrow \beta, t \rightarrow \infty$ for some $\beta \in \mathbf{R}$. Hence $\varphi(\Phi(t+r, r, x)) \rightarrow \beta, t \rightarrow \infty$, by the definition of $\psi$ and the properties of $\eta$. Hence $\varphi(y)=\beta$ for any $y \in \omega_{\Phi}(r, x)$. As any $y \in \omega_{\Phi}(r, x)$ lies on a full $\Theta$-orbit, the second assumption follows as well.

The following result may be more esthetically appealing.

Definition 2.6. An asymptotically autonomous semiflow $\Phi$ with limit semiflow $\boldsymbol{\theta}$ is called integrable asymptotically autonomous if, for any compact set $K$ in $X$, there exist some $s_{K}>0$ and an integrable function $\xi:\left[s_{K}, \infty\right) \rightarrow[0, \infty)$ such that

$$
\liminf _{t \searrow 0} \frac{1}{t} d(\Phi(t+s, s, y), \boldsymbol{\Theta}(t, y)) \leq \xi(s), \quad s \geq s_{K}, y \in K
$$

Theorem 2.7. Let $\Phi$ be an integrable asymptotically autonomous semiflow. Let the $\Phi$-orbit starting at the point $(r, x)$ be precompact, and let $\varphi$ be a Lyapunov function for the limit semiflow $\boldsymbol{\Theta}$ such that $\varphi$ is Lipschitz on compact subsets of $X$. Then there exists some $\beta \in \mathbf{R}$ such that $\varphi(y)=\beta$ for all $y \in \omega_{\Phi}(r, x)$. In particular, there exists a full $\Theta$-orbit $\theta$ through any $y \in \omega_{\Phi}(r, x)$ such that $\varphi(\theta(t))=\beta$ for all $t \in \mathbf{R}$.

Proof. Let $K$ be the closure of the forward $\Phi$-orbit starting at $(r, x)$. Let $\Lambda>0$ be the Lipschitz constant of $\varphi$ on $\Theta([0,1] \times K)$. Then, as $\varphi$ is a Lyapunov function for $\boldsymbol{\Theta}$, we have for $s \geq s_{K}, y \in K, 0 \leq t \leq 1$, that

$$
\begin{aligned}
\varphi(\Phi(t+s, s, y))-\varphi(y) & \leq \varphi(\Phi(t+s, s, y))-\varphi(\Theta(t, y)) \\
& \leq \Lambda d(\Phi(t+s, s, y), \boldsymbol{\Theta}(t, y)) .
\end{aligned}
$$

Hence, by Definition 2.6, for $s \geq s_{K}, y \in K$,

$$
\liminf _{t \searrow 0} \frac{1}{t}(\varphi(\Phi(t+s, s, y))-\varphi(y)) \leq \Lambda \xi(s) \text {. }
$$

Define

$$
\eta(s)=\Lambda \int_{s}^{\infty} \xi(\sigma) d \sigma
$$

and

$$
\psi(s, y)=\varphi(y)+\eta(s)
$$

Then

$$
\liminf _{t \searrow 0} \frac{1}{t}(\psi(\Psi(t,(s, y)))-\psi(s, y)) \leq 0, \quad s \geq s_{K}, y \in K
$$


Note that, for $t \geq s_{K}-r, \Psi(t,(r, x))$ is contained in $\left[s_{K}, \infty\right) \times K$. The semiflow property of $\Psi$ then implies that

$$
\liminf _{h \searrow 0} \frac{1}{h}(\psi(\Psi(t+h,(r, x)))-\psi(\Psi(t,(r, x)))) \leq 0
$$

for sufficiently large $t$. Hence $\psi(\Psi(t,(r, x)))$ is eventually decreasing in $t$ and thus has a limit $\beta$. By the definitions of $\Psi, \psi$, and $\eta, \varphi(\Phi(t+r, r, x))$ converges to $\beta$ as $t \rightarrow \infty$. Hence $\omega_{\Phi}(r, x)$ is contained in the $\beta$-level set of $\varphi$. In particular, there exists a full $\Theta$-orbit through any point of $\omega_{\Phi}(r, x)$ that is contained in the $\beta$-level set of $\varphi$.

\section{A CONVERSE TO THEOREM 1.8}

Consider the systems of ordinary differential equations

$$
y^{\prime}=g(y)
$$

on $\mathbf{R}^{n}$. We assume that $g$ is locally Lipschitz and that solutions of initial value problems for (3.1) exist for all future times. The notation $y \cdot t$ will be used for the solution of (3.1) starting at $y$ at $t=0$.

The main result of this section is a converse to Theorem 1.8; it says that every compact, connected, chain recurrent invariant set for (3.1) is the $\omega$-limit set of some asymptotically autonomous system with limit (3.1). The asymptotically autonomous system is of a particularly simple form.

Theorem 3.1. Let $A$ be a compact, connected, chain recurrent, invariant set for (3.1), and let $y_{0} \in A$. Then there exists a $C^{\infty}$ function $\psi:[0, \infty) \rightarrow \mathbf{R}^{n}$, such that $\psi(t) \rightarrow 0$ as $t \rightarrow \infty$ and $A$ is the $\omega$-limit set of the solution of

$$
y^{\prime}=g(y)+\psi(t), \quad y(0)=y_{0} .
$$

Moreover, for any $\varepsilon>0, \psi$ can be chosen such that $\psi(t) \leq \varepsilon, 0 \leq t<\infty$.

The following preliminary results are required in the proof of Theorem 3.1.

Let $\xi: \mathbf{R} \rightarrow \mathbf{R}$ be a $C^{\infty}$ function satisfying the following: $\xi(t)=0$ if and only if $t \notin(0,1), \xi(t)=1$ for $t \in(1 / 4,3 / 4), \xi(t)$ is increasing on $0<t<1 / 4$, and $\xi(t)$ is decreasing on $3 / 4<t<1$.

Consider the system

$$
y^{\prime}=g(y)+c \xi(t / \bar{t})
$$

where $c \in \mathbf{R}^{n}$ and $\bar{t}>0$. Let $y\left(t, y_{0}, c\right)$ denote the solution of (3.2) satisfying $y\left(0, y_{0}, c\right)=y_{0}$. (We will fix $\bar{t}$ in the following lemma so we do not include it in our notation.)

Lemma 3.2. Let $K \subset \mathbf{R}^{n}$ be compact and $\delta>0$. Then, there exists $\bar{t}>0$ such that $y\left(t, y_{0}, c\right)$ is uniquely defined on $0 \leq t \leq \bar{t}$, for all $y_{0} \in K$ and all $c \in \mathbf{R}^{n}$ satisfying $|c| \leq \delta$. There exist positive constants $k_{i}, i=1,2$, such that, for all $y_{0} \in K, c_{1}, c_{2}$ with $\left|c_{i}\right| \leq \delta$,

$$
k_{1}\left|c_{2}-c_{1}\right| \leq\left|y\left(\bar{t}, y_{0}, c_{2}\right)-y\left(\bar{t}, y_{0}, c_{1}\right)\right| \leq k_{2}\left|c_{2}-c_{1}\right| .
$$

Moreover, there exists some $\varepsilon>0$ such that, if $b \in \mathbf{R}^{n}$ satisfies

$$
\left|b-y_{0} \cdot \bar{t}\right|<\varepsilon,
$$


then there exists a unique $c=h\left(y_{0}, b\right)$ with $|c|<\delta$ satisfying

$$
y\left(\bar{t}, y_{0}, c\right)=b .
$$

Finally, $c=h\left(y_{0}, b\right)$ tends to zero as $b$ tends to $y_{0} \cdot \bar{t}$, uniformly in $y_{0} \in K$. In fact,

$$
k_{1}|c| \leq\left|b-y_{0} \cdot \bar{t}\right| \text {. }
$$

Proof. Standard existence results for ODEs imply that, given $\delta>0$, there exists $t_{1}>0$ such that for every $y_{0} \in K,|c| \leq \delta$, and $\bar{t}>0$ there exists a unique solution of (3.2) and $y(0)=y_{0}$, which we temporarily write as $y\left(t, y_{0}, c, \bar{t}\right)$, defined for $0 \leq t \leq t_{1}$. We stress that $t_{1}$ is independent of $\left(y_{0}, c, \bar{t}\right)$ restricted as above. Furthermore

$$
C=\left\{y: y=y\left(t, y_{0}, c, \bar{t}\right), y_{0} \in K,|c| \leq \delta, 0 \leq t \leq t_{1}, \bar{t}>0\right\}
$$

is bounded in $\mathbf{R}^{n}$. Let $L$ be a Lipschitz constant for $g$ on $C$ and fix $\bar{t} \leq t_{1}$ so that $L \bar{t} e^{L \bar{t}} \leq 1 / 4$. Hereafter, we drop $\bar{t}$ from the list of arguments of $y$. A Gronwall estimate gives

$$
\left|y\left(t, y_{0}, c_{2}\right)-y\left(t, y_{0}, c_{1}\right)\right| \leq \bar{t} e^{L \bar{t}}\left|c_{2}-c_{1}\right|, \quad 0 \leq t \leq \bar{t}
$$

Note that

$$
\gamma:=\int_{0}^{1} \xi(s) d s \in(1 / 2,1) .
$$

Integrating (3.2), we find that

$$
y\left(\bar{t}, y_{0}, c\right)=y_{0}+\int_{0}^{\bar{t}} g\left(y\left(s, y_{0}, c\right)\right) d s+\gamma c \bar{t} .
$$

From this and $\gamma>1 / 2$, we see that

$$
\begin{aligned}
(1 / 2) \bar{t}\left|c_{2}-c_{1}\right| \leq & \left|y\left(\bar{t}, y_{0}, c_{2}\right)-y\left(\bar{t}, y_{0}, c_{1}\right)\right| \\
& +\int_{0}^{\bar{t}} L\left|y\left(s, y_{0}, c_{2}\right)-y\left(s, y_{0}, c_{1}\right)\right| d s \\
\leq & \left|y\left(\bar{t}, y_{0}, c_{2}\right)-y\left(\bar{t}, y_{0}, c_{1}\right)\right|+\bar{t} L \bar{t} e^{L \bar{t}}\left|c_{2}-c_{1}\right| \\
\leq & \left|y\left(\bar{t}, y_{0}, c_{2}\right)-y\left(\bar{t}, y_{0}, c_{1}\right)\right|+(1 / 4) \bar{t}\left|c_{2}-c_{1}\right| .
\end{aligned}
$$

Consequently,

$$
(1 / 4) \bar{t}\left|c_{2}-c_{1}\right| \leq\left|y\left(\bar{t}, y_{0}, c_{2}\right)-y\left(\bar{t}, y_{0}, c_{1}\right)\right| \text {. }
$$

It follows that the map $c \mapsto y\left(\bar{t}, y_{0}, c\right)$ is injective and that its inverse $h$, defined by $b \mapsto c=h\left(y_{0}, b\right)$, is Lipschitz with Lipschitz constant $4 / \bar{t}$. Hence the image of $\{c:|c|<\delta\}$ under $y\left(\bar{t}, y_{0}, \cdot\right)$ is an open set containing a ball with center $y_{0} \cdot \bar{t}$ and radius $\varepsilon>0$. The other statements of the lemma follow with $k_{1}=\bar{t} / 4, k_{2}=\bar{t} e^{L \bar{t}}$.

The following lemma actually holds for autonomous semiflows in general.

Lemma 3.3. Let $T>0, \varepsilon>0$, and $y_{0} \in A$, where $A$ is as in Theorem 3.1. Then there exist sequences $\left\{y_{i}\right\}_{i \geq 1} \subset A,\left\{t_{i}\right\}_{i \geq 0}, t_{i}>T$, such that

$$
\left|y_{i} \cdot t_{i}-y_{i+1}\right|<\varepsilon, \quad i \geq 0, \quad\left|y_{i} \cdot t_{i}-y_{i+1}\right| \rightarrow 0, \quad i \rightarrow \infty .
$$

Furthermore, for each natural number $I, A$ is the closure of $\left\{y_{i}\right\}_{i \geq I}$. 
In other words, the $(\varepsilon, T)$-pseudo-orbit in $A$ starting at $y_{0}$, defined by

$$
y_{0} * t \equiv y_{i} \cdot\left(t-s_{i-1}\right), \quad s_{i-1} \leq t<s_{i},
$$

where $s_{i}=\sum_{n=0}^{i} t_{n}$, has $A$ as its $\omega$-limit set and its jumps converge to 0 as times tend to infinity.

Proof. This is a simple consequence of the compactness of $A$ and Lemma 1.4. For each natural number $n$, let $A_{n}$ be a finite subset of $A$ such that every point of $A$ is within $1 / n$ of a point of $A_{n}$. Now, starting at $y_{0}$, construct an $(\varepsilon, T)$-chain from $y_{0}$ to a point of $A_{1}$ and from that point to a different point of $A_{1}$, and so on until every point of $A_{1}$ has been visited. From the last point of $A_{1}$ visited, construct an $(\varepsilon / 2, T)$-chain from that point to a point in $A_{2}$ and continue constructing $(\varepsilon / 2, T)$-chains connecting points in $A_{2}$ until all points have been visited. Continuing in this way we obtain the desired infinite pseudo-orbit.

Proof of Theorem 3.1. Take $K=A$ in Lemma 3.2. Let $\bar{\xi}(t)=\xi(t / \bar{t})$. Let $T>2 \bar{t}, \varepsilon<k_{1}$, and $y_{0} * t$ be the infinite $(\varepsilon, T)$-pseudo-orbit given in Lemma 3.3. Define $\xi_{j}(t)=\bar{\xi}\left(t-s_{j}+\bar{t}\right)$ and

$$
\psi(t)=\sum_{j=0}^{\infty} c_{j} \xi_{j}(t)
$$

where

$$
c_{j}=h\left(y_{j} \cdot\left(t_{j}-\bar{t}\right), y_{j+1}\right) .
$$

Observe that the support of $\xi_{j}$ is $\left[s_{j}-\bar{t}, s_{j}\right]$ and therefore, since $t_{j}>T>2 \bar{t}$, the supports of $\xi_{j}$ and $\xi_{k}$ do not intersect for $j \neq k$. Furthermore, as

$$
\left|y_{j} \cdot t_{j}-y_{j+1}\right|<\varepsilon<k_{1},
$$

$c_{j}$ is well defined by Lemma 3.2. Since $y_{j} \cdot t_{j}-y_{j+1} \rightarrow 0$ as $j \rightarrow \infty$ and

$$
k_{1}\left|c_{j}\right| \leq\left|y_{j} \cdot t_{j}-y_{j+1}\right|,
$$

we conclude that $c_{j} \rightarrow 0$ as $j \rightarrow \infty$ and, in particular, it follows that $\psi(t) \rightarrow 0$ as $t \rightarrow \infty$. Let $y(t)$ be the solution of

$$
y^{\prime}=g(y)+\psi(t), \quad y(0)=y_{0} .
$$

Then $y(t)=y_{0} * t$ except for $t$ in the intervals $\left(s_{j}-\bar{t}, s_{j}\right)$ where $y(t)=$ $y\left(t-s_{j}+\bar{t}, y_{j} \cdot\left(t_{j}-\bar{t}\right), c_{j}\right)$ and $y\left(t, y_{0}, c\right)$ is as in Lemma 3.2. $y_{0} * t$ is the pseudo-orbit obtained in Lemma 3.3. Since $\psi(t) \rightarrow 0, t \rightarrow \infty$, and the solutions of ODEs depend continuously on their (locally Lipschitz continuous) vector fields (see [H1]), we have that $\left|y(t)-y_{0} * t\right| \rightarrow 0, t \rightarrow \infty$. In particular $y$ has the same $\omega$-limit set as the pseudo-orbit $y_{0} * t$ which is the set $A$ by Lemma 3.3.

As an example, consider the case that (3.1) is the Duffing equation

$$
y_{1}^{\prime}=y_{2}, \quad y_{2}^{\prime}=y_{1}-y_{1}^{3} .
$$

The function $\varphi(y)=y_{2}^{2} / 2-y_{1}^{2} / 2+y_{1}^{4} / 4$ is constant along trajectories of (3.3). If $-1 / 4 \leq a \leq b \leq \infty$, then any connected component, $A$, of $\varphi^{-1}([a, b])$ is a compact, connected, chain recurrent, invariant set for (3.3). Consequently, 
by Theorem 3.1, given $\left(\bar{y}_{1}, \bar{y}_{2}\right) \in A$, there exists a smooth function $\xi(t)=$ $\left(\xi_{1}(t), \xi_{2}(t)\right)$ on $t \geq 0$, satisfying $\xi_{i}(t) \rightarrow 0$ as $t \rightarrow \infty$ and such that the $\omega$-limit set of the solution of

$$
x_{1}^{\prime}=x_{2}+\xi_{1}(t), \quad x_{2}^{\prime}=x_{1}-x_{1}^{3}+\xi_{2}(t)
$$

satisfying $x_{1}(0)=\bar{y}_{1}, x_{2}(0)=\bar{y}_{2}$ is $A$. This example also illustrates the fact that Theorem 2.2. is sharp.

We mention that, if $a<b$, the functions $\xi_{1}, \xi_{2}$ cannot both be chosen to be integrable. Otherwise Theorem 2.7 would apply and the $\omega$-limit set would be contained in a level set of $\varphi$.

Theorem 3.1, in connection with the description of $\omega$-limit sets of asymptotically autonomous semiflows in [T1, Theorem 4.2], can be used to describe chain recurrent sets for (3.1).

Theorem 3.4. Let $g$ be continuously differentiable on $\mathbf{R}^{n}$ and $A$ be a compact, connected, chain recurrent, invariant set for (3.1). Assume that the $\omega$-limit set of any forward bounded solution of (3.1) contains a critical point and that all critical points of (3.1) are hyperbolic. Then $A$ consists of exactly one critical point or it contains a cyclic chain of orbits of (3.1) that connect critical points.

The assumption that all critical points are hyperbolic can be replaced by the more general assumption that all critical points are isolated compact invariant sets for (3.1), notably when $g$ is only locally Lipschitz rather than continuously differentiable. In the plane a more precise description of $A$ is possible (see [T2, Theorem 1.5]).

\section{REFERENCES}

[B] J. M. Ball, On the asymptotic behavior of generalized processes, with applications to nonlinear evolution equations, J. Differential Equations 27 (1978), 224-265.

[C1] C. Conley, The gradient structure of a flow: I, IBM Research, RC 3939 (\#17806), Yorktown Heights, NJ, July 17, 1972; Ergodic Theory Dynamical Systems 8 (1988), 11-26.

[C2] _- Isolated invariant sets and the Morse index, CBMS Regional Conf. Ser. in Math., no. 38, Amer. Math. Soc., Providence, RI, 1978.

[D] C. M. Dafermos, Semiflows associated with compact and uniform processes, Math. Systems Theory 8 (1975), 142-149.

[FS] J. Frank and J. Selgrade, Abstract $\omega$-limit sets, chain recurrent sets, and basic sets for flows, Proc. Amer. Math. Soc. 60 (1976), 309-316.

[H1] J. K. Hale, Ordinary differential equations, Krieger, Malabar, FL, 1980.

[H2] - Asymptotic behavior of dissipative systems, Math. Surveys Monographs, vol. 25, Amer. Math. Soc., Providence, RI, 1988.

[HV] J. K. Hale and S. M. Verduyn Lunel, Introduction to functional differential equations, Springer-Verlag, New York, 1993.

[L] J. P. LaSalle, Stability theory and invariance properties, Dynamical Systems, Vol. 1 (L. Cesari, J. Hale, and J. LaSalle, eds.), Academic Press, 1976, pp. 211-222.

[Ma] L. Markus, Asymptotically autonomous differential systems, Contributions to the Theory of Nonlinear Oscillations 111 (S. Lefschetz, ed.), Ann. of Math. Stud., no. 36, Princeton Univ. Press, Princeton, NJ, 1956, pp. 17-29.

[Mi] R. K. Miller, Almost periodic differential equations as dynamical systems with applications to the existence of a.p. solutions, J. Differential Equations 1 (1965), 337-345.

[MS1] R. K. Miller and G. Sell, A note on Volterra integral equations and topological dynamics, Bull. Amer. Math. Soc. 74 (1968), 904-908. 
[MS2] _ Volterra integral equations and topological dynamics, Mem. Amer. Math. Soc., No. 102 (1970).

[NS] V. V. Nemytskii and V. V. Stepanov, Qualitative theory of differential equations, Princeton Univ. Press, Princeton, NJ, 1960.

[R] C. Robinson, Stability theorems and hyperbolicity in dynamical systems, Rocky Mountain J. Math. 7 (1977), 425-437.

[Sa] S. H. Saperstone, Semidynamical systems in infinite dimensional spaces, Springer-Verlag, New York, 1981.

[Se1] G. Sell, Nonautonomous differential equations and topological dynamics. I. The basic theory, Trans. Amer. Math. Soc. 127 (1967), 241-262.

[Se2] _ Nonautonomous differential equations and topological dynamics. II. Limiting equations, Trans. Amer. Math. Soc. 127 (1967), 263-283.

[T1] H. R. Thieme, Convergence results and a Poincaré-Bendixson trichotomy for asymptotically autonomous differential equations, J. Math. Biol. 30 (1992), 755-763.

[T2] - Asymptotically autonomous differential equations in the plane, Rocky Mountain J. Math. 24 (1994), 351-380.

(K. Mischaikow) Center for Dynamical Systems and Nonlinear Studies, School of Mathematics, Georgia Institute of Technology, Atlanta, Georgia 30332

(H. Smith and H. Thieme) Department of Mathematics, Arizona State University, TEMPE, ARIZONA 85287-1804

E-mail address: halsmith@math.la.asu.edu

E-mail address: athrtcasurm.inre.asu.edu 